\title{
The ultrametric corona problem
}

\section{by Alain Escassut and Nicolas Maïnetti}

\begin{abstract}
Let $K$ be a complete ultrametric algebraically closed field and let $A$ be the Banach $K$-algebra of bounded analytic functions in the "open" unit disk $D$ of $K$ provided with the Gauss norm. Let $\operatorname{Mult}(A, \|$. $\|)$ be the set of continuous multiplicative seminorms of $A$ provided with the topology of simple convergence, let $\operatorname{Mult}_{m}(A, \|$. \|) be the subset of the $\phi \in \operatorname{Mult}\left(A, \|\right.$. \|) whose kernel is a maximal ideal and let $\operatorname{Mult}_{a}(A, \|$. $\|)$ be the subset of the $\phi \in \operatorname{Mult}(A, \|$. $\|)$ whose kernel is a maximal ideal of codimension 1 . For every maximal ideal $\mathcal{M}$, there exist ultrafilters $\mathcal{U}$ on $D$ such that $\mathcal{M}$ is the set of functions $f \in A$ vanishing along $\mathcal{U}$ and there exists at least one $\psi \in \operatorname{Mult}_{m}(A, \|$. $\|)$ of kernel $\mathcal{M}$ equal to a limit of $|f(x)|$ along $\mathcal{U}$. Certain ultrafilters define a unique $\psi \in \operatorname{Mult}_{m}(A, \|$. $\|)$. If every maximal ideal is the kernel of only one $\psi \in \operatorname{Mult}_{m}(A,\|\|$.$) , then \operatorname{Mult}_{a}(A,\|\|$. is dense in $\operatorname{Mult}_{m}(A, \|$. $\|)$. This is the case when $K$ is strongly valued or spherically complete. Given a continuous multiplicative norm $\psi$ on $A$ other than the Gauss norm, $\psi$ is defined by a circular filter on $D$ of diameter $r<1$. If $K$ is of characteristic zero, the algebra $A$ admits proper closed prime ideals that are neither zero nor maximal ideals.
\end{abstract}

2000 Mathematics subject classification: Primary 12J25 Secondary 46S10

\section{Introduction.}

Let $K$ be an algebraically closed field complete with respect to an ultrametric absolute value $\mid$. $\mid$. Given $a \in K$ and $r>0$, we denote by $d(a, r)$ the disk $\{x \in K|| x-a \mid \leq r\}$, by $d\left(a, r^{-}\right)$the disk $\{x \in K|| x-a \mid<r\}$, by $C(a, r)$ the circle $\{x \in K|| x-a \mid=r\}$ and set $D=d\left(0,1^{-}\right)$. Let $A$ be the $K$-algebra of bounded power series converging in $D$ which is complete with respect to the Gauss norm defined as $\left\|\sum_{n=1}^{\infty} a_{n} x^{n}\right\|=\sup _{n \in \mathbb{N}}\left|a_{n}\right|$ : we know that this norm actually is the norm of uniform convergence on $D[6],[16]$.

In [19] the Corona problem was considered in a similar way as it is on the field $\mathbb{C}[3]$, [15]: the author asked the question whether the set of maximal ideals of $A$ defined by the points of $D$ (which are well known to be of the form $(x-a) A$ ) is dense in the whole set of maximal ideals with respect to the so-called "Gelfand Topology". In fact, this makes no sense because the maximal ideals which are not of the form $(x-a) A$ are of infinite codimension [11]. Consequently, a Corona problem should be defined in a different way.

Given a commutative $K$-algebra $B$ with unity, provided with a $K$-algebra norm $\|$. $\|$, the set of continuous multiplicative $K$-algebra semi-norms of $B$ was studied in many works [1], [5], [6], [9] and is usually denoted by $\operatorname{Mult}(B, \|$. \|) [5], [6], [9]. For each $\phi \in \operatorname{Mult}(B, \|$. $\|)$, we denote by $\operatorname{Ker}(\phi)$ the closed prime ideal of the $f \in B$ such that $\phi(f)=0$. The set of the $\phi \in \operatorname{Mult}(B,\|\|$.$) such that \operatorname{Ker}(\phi)$ is a maximal ideal is denoted by $\operatorname{Mult}_{m}(B,\|\|$.$) , the set of the \phi \in \operatorname{Mult}(B, \|$. \|) such that $\operatorname{Ker}(\phi)$ is a maximal ideal of codimension 1 is denoted by $\operatorname{Mult}_{a}(B,\|\|$.$) and here, the set of the continuous$ multiplicative norms of $A$ will be denoted by $\operatorname{Mult}_{0}(B,\|\|$.$) .$ 
We know that $\sup \{\phi(f) \mid \phi \in \operatorname{Mult}(B,\|\|)\}=.\lim _{n \rightarrow \infty}\left(\left\|f^{n}\right\|\right)^{\frac{1}{n}} \forall f \in B$ [7], [8], [13], [14]. On the other hand, $\operatorname{Mult}(B, \|$. $\|)$ is provided with the topology of simple convergence and is compact in this topology.

We know that for every $\mathcal{M} \in \operatorname{Max}(B)$, there exists at least one $\phi \in \operatorname{Mult}_{m}(B,\|\|$. such that $\operatorname{Ker}(\phi)=\mathcal{M}$ but in certain cases, there exist infinitely many $\phi \in \operatorname{Mult}_{m}(B,\|\|$. such that $\operatorname{Ker}(\phi)=\mathcal{M}$ [4], [7], [8]. A maximal ideal $\mathcal{M}$ of $B$ is said to be univalent if there is only one $\phi \in \operatorname{Mult}_{m}(B, \|$. $\|)$ such that $\operatorname{Ker}(\phi)=\mathcal{M}$ and the algebra $B$ is said to be multbijective if every maximal ideal is univalent (so, non-multbijective commutative Banach $K$-algebras with unity do exist).

Thus, the ultrametric Corona problem may be viewed at two levels:

1) Is $\operatorname{Mult}_{a}(A, \|$. $\|)$ dense in $\operatorname{Mult}_{m}(A, \|$. $\|$ ) (with respect to the topology of simple convergence)?

2) Is $\operatorname{Mult}_{a}(A,\|\|$.$) dense in \operatorname{Mult}(A,\|\|$.$) (with respect to the same topology )?$

Actually, this way to set the Corona problem on an ultrametric field is not really different from the original problem once considered on $\mathbb{C}$ because on a commutative $\mathbb{C}$ Banach algebra with unity, all continuous multiplicative semi-norms are known to be of the form $|\chi|$ where $\chi$ is a character of $A$. Thus the Corona problem was equivalent to show that the set of multiplicative semi-norms defined by points of the open disk was dense inside the whole set of continuous multiplicative semi-norms, with respect to the topology of simple convergence.

Remark: Given a filter $\mathcal{G}$, if for every $f \in A,|f(x)|$ admits a limit $\varphi_{\mathcal{G}}(f)$ along $\mathcal{G}$, the function $\varphi_{\mathcal{G}}$ obviously belongs to $\operatorname{Mult}(A,\|\|$.$) . Moreover, it clearly lies in the closure of$ $\operatorname{Mult}_{a}(A,\|\|$.$) . Consequently, if we can prove that every element of \operatorname{Mult}_{m}(A, \|$. $\|)$ is of the form $\varphi_{\mathcal{G}}$, with $\mathcal{G}$ a certain filter on $D$, Question 1) is solved. And similarly, if we could prove that every element of $\operatorname{Mult}(A,\|\|$.$) is of the form \varphi_{\mathcal{G}}$, Question 2) would be solved.

Studying such problems first requires to know the nature of continuous multiplicative semi-norms on $A$.

Definitions and notation: Let $a \in D$ and let $R \in] 0,1]$. Given $r, s \in \mathbb{R}$ such that $0<r<s$ we set $\Gamma(a, r, s)=\{x \in K|r<| x-a \mid<s\}$.

We call circular filter of center $a$ and diameter $R$ on $D$ the filter $\mathcal{F}$ which admits as a generating system the family of sets $\Gamma\left(\alpha, r^{\prime}, r^{\prime \prime}\right) \cap D$ with $\alpha \in d(a, R), r^{\prime}<R<r^{\prime \prime}$, i.e. $\mathcal{F}$ is the filter which admits for basis the family of sets of the form $D \cap\left(\bigcap_{i=1}^{q} \Gamma\left(\alpha_{i}, r_{i}^{\prime}, r_{i}^{\prime \prime}\right)\right)$ with $\alpha_{i} \in d(a, R), r_{i}^{\prime}<R<r_{i}^{\prime \prime} \quad(1 \leq i \leq q, q \in \mathbb{N})$.

Recall that the field $K$ is said to be spherically complete if every decreasing sequence of disks has a non-empty intersection (it is known that $\mathbb{C}_{p}$ is not spherically complete but it has a spherical completion).

In a field which is not spherically complete, one has to consider decreasing sequences of disks $\left(D_{n}\right)$ with an empty intersection. We call circular filter with no center, of canonical basis $\left(D_{n}\right)$ the filter admitting for basis the sequence $\left(D_{n}\right)$ and the number $\lim _{n \rightarrow \infty} \operatorname{diam}\left(D_{n}\right)$ is called diameter of the filter. 
Finally the filter of neighborhoods of a point $a \in D$ is called circular filter of the neighbourhoods of a on $D$ and its diameter is 0 . Given a circular filter $\mathcal{F}$, its diameter is denoted by $\operatorname{diam}(\mathcal{F})$.

Here, we shall denote by $\mathcal{W}$ the circular filter on $D$ of center 0 and diameter 1 . A filter $\mathcal{F}$ on $D$ will be called a coroner filter if it is thinner than $\mathcal{W}$. Similarly, a sequence $\left(a_{n}\right)$ on $D$ will be called a coroner sequence if its filter is a coroner filter, i.e. if $\lim _{n \rightarrow+\infty}\left|a_{n}\right|=1$. An ultrafilter $\mathcal{U}$ on $D$ will be called coroner ultrafilter if it is thinner than $\mathcal{W}$.

Let $\psi \in \operatorname{Mult}(A,\|\|$.$) . Then \psi$ is said to be coroner if its restriction to $K[x]$ equal to $\|$.$\| .$

Let $\left(a_{n}\right)_{n \in \mathbb{N}}$ be a coroner sequence in $D$. The sequence is called a regular sequence if $\inf _{j \in \mathbb{N}} \prod_{\substack{n \in \mathbb{N} \\ n \neq j}}\left|a_{n}-a_{j}\right|>0$.

An ultrafilter $\mathcal{U}$ is said to be regular if it is thinner than a regular sequence. Thus, by definition, a regular ultrafilter is a coroner ultrafilter.

Two coroner ultrafilters $\mathcal{F}, \mathcal{G}$ are said to be contiguous if for every subsets $F \in \mathcal{F}, G \in$ $\mathcal{G}$ of $D$ the distance from $F$ to $G$ is null.

Let $f \in A$. Recall that $f$ is said to be quasi-invertible if it factorizes in $A$ in the form $f=P g$ with $P \in K[x]$ and $g$ an invertible element of $A$.

On $K[x]$, circular filters on $K$ are known to characterize multiplicative semi-norms by associating to each circular filter $\mathcal{F}$ the multiplicative semi-norm $\varphi_{\mathcal{F}}$ defined as $\varphi_{\mathcal{F}}(f)=$ $\lim _{\mathcal{F}}|f(x)|[5],[6],[7],[12]$.

We know that every $f \in A$ is an analytic element in each disk $d(a, r)$ whenever $a \in D, r \in] 0,1[$ [6]. Consequently, by classical results [6], several properties of polynomials have continuation to $A$.

Remark: A regular sequence is an idempotent polar sequence which and is not a $T$ polar sequence [18]. However, a polar sequence which and is not a $T$-polar sequence is not necessarily a regular sequence.

The paper is aimed at showing that in at least when the field $K$ satisfies certain hypotheses, $A$ is multbijective and therefore $\operatorname{Mult}_{a}(A, \|$. $\|)$ is dense in $\operatorname{Mult}_{m}(A, \|$. $\|)$. On the other hand, we will examine relations between closed prime ideals, maximal ideals and multiplicative semi-norms.

\section{Basic results}

Describing problems and results requires to state a lot of basic results (some of them are well known by specialists). Theorems 1, 2, 3 are immediate:

Theorem 1: For every $f \in A,\|f\|=\lim _{\mathcal{Y}}|f(x)|$.

Theorem 2: Every element of $A$ is uniformly continuous in D. 
Corollary 2.1: Let $\mathcal{U}_{1}, \mathcal{U}_{2}$ be two coroner contiguous ultrafilters on D. Then $\mathcal{J}\left(\mathcal{U}_{1}\right)=$ $\mathcal{J}\left(\mathcal{U}_{2}\right)$.

Remark: An interesting question is whether two coroner ultrafilters $\mathcal{U}_{1}$ and $\mathcal{U}_{2}$ such that $\mathcal{J}\left(\mathcal{U}_{1}\right)=\mathcal{J}\left(\mathcal{U}_{2}\right)$ are contiguous. The problem is partially solved in Theorem 24.

Theorem 3: Let $\mathcal{U}$ be an ultrafilter on D. For every $f \in A,|f(x)|$ admits a limit $\varphi_{\mathcal{U}}(f)$ along $\mathcal{U}$. Moreover, the mapping $\varphi_{\mathcal{U}}$ from $A$ to $\mathbb{R}_{+}$belongs to $\operatorname{Mult}(A,\|\|$.$) and$ $\operatorname{Ker}(\varphi \mathcal{U})=\mathcal{J}(\mathcal{U})$. Given two contiguous ultrafilters $\mathcal{U}_{1}, \mathcal{U}_{2}$ on $D, \varphi_{\mathcal{U}_{1}}=\varphi_{\mathcal{U}_{2}}$.

Theorem 4: For every circular filter $\mathcal{F}$ secant with $D$, of diameter $r<1, \varphi_{\mathcal{F}}$ has continuation to an element of $\operatorname{Mult}(A,\|\|$.$) .$

Theorem 5 also is classical [9]:

Theorem 5: An element $f \in A$ is not quasi-invertible if and only if it has infinitely many zeros. If $f$ is not quasi-invertible, its set of zeros is a coroner sequence $\left(a_{n}\right)$.

Theorems 6, 7 and 9, 10, 11, 12 may be found in [6].

Theorem 6: Let I be an ideal of $A$. The following two statements are equivalent:

i) I is generated by a polynomial whose zeros lie in $D$,

ii) I contains a quasi-invertible element.

Theorem 7: Let $\mathcal{M}$ be a principal maximal ideal of $A$. Then there exists $a \in D$ such that $\mathcal{M}=(x-a) A$.

Concerning maximal ideals, Theorem 8 is proved in [9].

Theorem 8: Let $\mathcal{M}$ be a non-principal maximal ideal of $A$. Then $\mathcal{M}$ is not of finite type.

Corollary 8.1: The mapping from $D$ to $\operatorname{Max}(A)$ associating to each point a of $D$ the maximal ideal $(x-a) A$ is a bijection from $D$ onto the set of principal maximal ideals.

Theorem 9: $\quad$ Non-principal maximal ideals of $A$ are of infinite codimension. [9]

Theorem 10: Let $\mathcal{M}$ be a maximal ideal of $A$. The following statements are equivalent:

i) $\mathcal{M}$ is of finite type,

ii) $\mathcal{M}$ is principal,

iii) there exists $a \in D$ such that $\mathcal{M}=(x-a) A$

iv) $\mathcal{M}$ is of codimension 1.

Corollary 10.1: An element $\phi$ of $\operatorname{Mult}(A,\|\|$.$) belongs to \operatorname{Mult}_{a}(A,\|\|$.$) if and only$ if there exists $a \in D$ such that $\phi(f)=|f(a)|, \forall f \in A$. 
In order to understand the way to prove the main theorems, it is indispensable to define sets $\mathcal{D}(h, \epsilon)$.

Notation: Let $h \in A, \epsilon>0$. We set $\mathcal{D}(h, \epsilon)=\{x \in D|| h(x) \mid \leq \epsilon\}$.

Theorem 11: Let $\mathcal{P}$ be a prime non-principal ideal of $A$. Let $r \in] 0,1[$ and $\epsilon \in] 0,1[$. There exists $h \in \mathcal{P}$ such that $\mathcal{D}(h, \epsilon) \subset \Gamma(0, r, 1)$.

Theorem 12: $\quad$ Let $\mathcal{M}$ be a non-principal maximal ideal of $A$ and let $\psi \in \operatorname{Mult}_{m}(A, \|$. $\|)$ satisfy $\operatorname{Ker}(\psi)=\mathcal{M}$. Every quasi-invertible element $f \in A$ satisfies $\psi(f)=\lim _{\mathcal{W}}|f(x)|=\|f\|$.

Theorem 13 is shown in [19] and is a Bezout-like theorem:

Theorem 13: Let $f_{1}, \ldots, f_{q} \in A$ satisfy $\left\|f_{j}\right\|<1 \forall j=1, \ldots, q$ and $\inf \left\{\max _{j=1, \ldots, q}\left(\left|f_{j}(x)\right|\right) \mid x \in D\right\}=\omega>0$. There exist $g_{1}, \ldots, g_{q} \in A$ such that $\sum_{j=1}^{q} g_{j} f_{j}=1$ and $\max _{j=1, \ldots, q}\left\|g_{j}\right\|<\omega^{-2}$.

Corollary 13.1: Let I be an ideal of $A$ different from $A$. The family of sets $\mathcal{D}(f, \epsilon), f \in$ $I, \epsilon>0$, makes a system of generators of a filter on $D$.

Notation: Let $I$ be an ideal of $A$ different from $A$. We will denote by $\mathcal{G}_{I}$ the filter generated by the sets $\mathcal{D}(f, \epsilon), f \in I, \epsilon>0$. By definition, $\mathcal{G}_{I}$ is minimal, with respect to the relation of thinness, among the filters $\mathcal{H}$ such that $\lim _{\mathcal{H}} f(x)=0 \forall f \in I$.

As a corollary of Theorem 11 and Corollary 13.1, we have Corollary 13.2

Corollary 13.2: Let $\mathcal{P}$ be a non-principal prime ideal of $A$. Then $\mathcal{G}_{\mathcal{P}}$ is coroner.

Corollary 13.3: Let $\mathcal{M}$ be a non-principal maximal ideal of $A$. Then $\mathcal{G}_{\mathcal{M}}$ is coroner and $\mathcal{M}=\mathcal{J}\left(\mathcal{G}_{\mathcal{M}}\right)$. Moreover, for every ultrafilter $\mathcal{U}$ thinner than $\mathcal{G}_{\mathcal{M}}$, then $\mathcal{J}(\mathcal{U})=\mathcal{M}$.

Corollary 13.4: $\quad$ Let $\mathcal{M}$ be a non-principal maximal ideal of $A$ and let $\mathcal{U}$ be an ultrafilter thinner than $\mathcal{G}_{\mathcal{M}}$. Then $\varphi_{\mathcal{U}}$ belongs to the closure of $\operatorname{Mult}_{a}(A,\|\|$.$) in \operatorname{Mult}_{m}(A, \|$. $\|)$.

Corollary 13.5: Let $\mathcal{M}$ be a univalent non-principal maximal ideal of $A$ and let $\phi \in$ $\operatorname{Mult}_{m}(A, \|$. $\|)$ satisfy $\operatorname{Ker}(\phi)=\mathcal{M}$. Then $\phi$ is of the form $\phi(f)=\lim _{\mathcal{U}}|f(x)|$ with $\mathcal{U}$ a coroner ultrafilter such that $\mathcal{J}(\mathcal{U})=\mathcal{M}$. Moreover, $\phi$ belongs to the closure of $\operatorname{Mult}_{a}\left(A, \|\right.$. \|) in $\operatorname{Mult}_{m}(A,\|\|$.$) .$

Corollary 13.6: Suppose $A$ is multbijective. Then every multiplicative semi-norm $\phi \in$ $\operatorname{Mult}_{m}(A, \|$. $\|) \backslash \operatorname{Mult}_{a}(A, \|$. $\|)$ is of the form $\phi(f)=\lim _{\mathcal{U}}|f(x)|$ with $\mathcal{U}$ a coroner ultrafilter such that $\mathcal{J}(\mathcal{U})=\mathcal{M}$. Moreover, $\operatorname{Mult}_{a}\left(A, \|\right.$. \|) is dense in $\operatorname{Mult}_{m}(A, \|$. $\|)$. 
Definition: The field $K$ is said to be strongly valued if at least one of the following sets is not countable: the set of values of $K=\{|x| \mid x \in K\}$ and the residue class field of $K$.

Let us recall the following Theorem [5], [7]:

Theorem 14: Suppose $K$ is strongly valued. Every commutative $K$-Banach algebra is multbijective.

Corollary 14.1: Suppose $K$ is strongly valued. Then every multiplicative semi-norm $\phi \in \operatorname{Mult}_{m}(A,\|\|.) \backslash \operatorname{Mult}_{a}\left(A, \|\right.$. \|) is of the form $\phi(f)=\lim _{\mathcal{U}}|f(x)|$ with $\mathcal{U}$ a coroner ultrafilter such that $\mathcal{J}(\mathcal{U})=\mathcal{M}$. Moreover, $\operatorname{Mult}_{a}\left(A, \|\right.$. \|) is dense in $\operatorname{Mult}_{m}(A, \|$. \|).

\section{Multbijectivity in a spherically complete field}

Theorem 18 is proved with help of Propositions $15,16,17$. The hypothesis $K$ spherically complete is essential in Proposition 17 because if the field $K$ is not spherically complete, we can't factorize $h$ as done in Proposition 16.

Proposition 15: Let $(B,\|\|$.$) be a commutative ultrametric K$-Banach algebra with unity. Suppose there exist $f \in B, \phi, \psi \in \operatorname{Mult}(B, \|$. $\|)$ such that $\psi(f)<\phi(f), s p(f) \cap$ $\Gamma(0, \psi(f), \phi(f))=\emptyset$ and there exists $\epsilon \in] 0, \phi(f)-\psi(f)\left[\right.$ satisfying further $\left\|(f-a)^{-1}\right\| \leq$ $M \forall a \in \Gamma(0, \psi(f), \phi(f)-\epsilon)$. Then there exists $\gamma \in B$ such that $\psi(\gamma)=1, \phi(\gamma)=0$.

Proposition 16: Let $\mathcal{M}$ be a non-principal maximal ideal of $A$ and let $\mathcal{U}$ be an ultrafilter on $D$ such that $\mathcal{M}=\mathcal{J}(\mathcal{U})$. Let $f \in A \backslash \mathcal{M}$ be not invertible in $A$ and let $g \in A, h \in \mathcal{M}$ such that $f g=1+h$. Let $\lambda=\varphi_{\mathcal{U}}(f)$, let $\left.\epsilon \in\right] 0, \min (\lambda, 1)[$ and let $\Lambda=\left\{x \in D|| f(x) g(x)|-1|_{\infty}<\epsilon,|| f(x)|-\lambda|_{\infty}<\epsilon\right\}$.

Suppose that there exist a function $\bar{h} \in A$ admitting for zeroes in $D$ the zeroes of $h$ in $D \backslash \Lambda$ and a function $\widehat{h} \in A$ admitting for zeroes the zeroes of $h$ in $\Lambda$, each counting multiplicities, so that $h=\bar{h} \widehat{h}$. Then $|\bar{h}(x)|$ has a strictly positive lower bound in $\Lambda$ and $\widehat{h}$ belongs to $\mathcal{M}$.

Moreover, there exists $\omega \in] 0, \lambda[$ such that $\omega \leq \inf \{\max (|f(x)|,|\widehat{h}(x)|) \mid x \in D\}$. Further, for every $a \in d(0,(\lambda-\epsilon))$, we have $\omega \leq \inf \{\max (|f(x)-a|,|\widehat{h}(x)|) \mid x \in D\}$.

Proposition 17: $\quad$ Suppose $K$ is spherically complete. Let $\mathcal{M}$ be a non-principal maximal ideal of $A$ and let $\mathcal{U}$ be an ultrafilter on $D$ such that $\mathcal{M}=\mathcal{J}(\mathcal{U})$. Let $f \in A \backslash \mathcal{M}$ satisfy $\|f\|<1$, let $\lambda=\varphi_{\mathcal{U}}(f)$ and let $\left.\epsilon \in\right] 0, \lambda[$. There exists $\omega>0$ such that, for every $a \in$ $d(0, \lambda-\epsilon)$, there exists $g_{a} \in A$ satisfying $(f-a) g_{a}-1 \in \mathcal{M}$ and $\left\|g_{a}\right\| \leq \omega^{-2}$.

Now, we can conclude when $K$ is spherically complete:

Theorem 18: If $K$ is spherically complete, then $A$ is multbijective.

The proof of Theorem 18 consists of assuming that there exists $\psi, \phi \in \operatorname{Mult}_{m}(A, \|$. $\|)$ satisfying $\psi(P)=\phi(P)=\|P\| \forall P \in K[x] \operatorname{Ker}(\psi)=\operatorname{Ker}(\phi)$ and $\psi(f)<\phi(f)$ for certain $f \in A$. Using the ultrametric holomorphic functional calculus and Propositions 15, 16, 17 we can construct a function $g$ satisfying $\psi(g)=0, \phi(g)>0$, a contradiction. 
Corollary 18.1: If $K$ is spherically complete, then for every $\phi \in \operatorname{Mult}_{m}(A, \|$. $\|)$ there exists a coroner ultrafilter $\mathcal{U}$ such that $\phi=\varphi_{\mathcal{U}}$.

Corollary 18.2: If $K$ is spherically complete, then $\operatorname{Mult}_{a}(A,\|\|$.$) is dense in \operatorname{Mult}_{m}(A,\|\|$.$) .$

\section{Regular maximal ideals}

Regular coroner ultrafilters give a very nice representation of certain maximal ideals. We will first recall links with bounded sequences [19].

Definitions and notation: Let $\mathcal{B}(\mathbb{N}, K)$ be the $K$-Banach algebra of bounded sequences of $K$ provided with the usual laws of $K$-algebra and with the usual norm defined as $\left\|\left(a_{n}\right)_{n \in \mathbb{N}}\right\|^{\prime}=\sup \left\{\left|a_{n}\right| \mid n \in \mathbb{N}\right\}$. For every ultrafilter $\mathcal{G}$ on $\mathbb{N}$ we will denote by $\Theta(\mathcal{G})$ the ideal of $\mathcal{B}(\mathbb{N}, K)$ consisting of sequences $\left(a_{n}\right)$ such that $\lim _{\mathcal{G}} a_{n}=0$.

Let $\mathcal{S}=\left(a_{n}\right)_{n \in \mathbb{N}}$ be a coroner sequence. We will denote by $\Sigma(\mathcal{S})$ the set of ultrafilters thinner than $\mathcal{S}$, by $\mathcal{I}(\mathcal{S})$ the ideal of the $f \in A$ such that $f\left(a_{n}\right)=0 \forall n \in \mathbb{N}$.

Recall that an ultrafilter $\mathcal{G}$ is said to be principal if $\bigcap_{F \in \mathcal{G}} F$ is a singleton.

Theorem 19 is classical:

Theorem 19: $\Theta$ is a bijection from the set of ultrafilters on $\mathbb{N}$ onto $\operatorname{Max}(\mathcal{B}(\mathbb{N}, K))$. The restriction of $\Theta$ to the subset of non-principal ultrafilters on $\mathbb{N}$ is a bijection from this set onto the set of non-principal maximal ideals of $\mathcal{B}(\mathbb{N}, K)$. Moreover, a maximal ideal of $\mathcal{B}(\mathbb{N}, K)$ is principal if and only it is of codimension 1.

Theorem 20: Let $M$ be a non-principal maximal ideal of $\mathcal{B}(\mathbb{N}, K)$ and let $U=\Theta^{-1}(M)$. Let $\theta$ be the canonical surjection from $\mathcal{B}(\mathbb{N}, K)$ onto the field $L=\frac{\mathcal{B}(\mathbb{N}, K)}{M}$. Let \|\|$_{M}$ be the $K$-algebra quotient norm of $L$. Then, every sequence $\left(a_{n}\right)_{n \in \mathbb{N}} \in \mathcal{B}(\mathbb{N}, K)$ satisfies $\left\|\left(a_{n}\right)\right\|_{M}=\lim _{U}\left|a_{n}\right|$.

Corollary 20.1: $\mathcal{B}(\mathbb{N}, K)$ is multbijective. Let $M$ be a principal maximal ideal of $\mathcal{B}(\mathbb{N}, K)$. The $K$-Banach algebra quotient norm of the field $\frac{\mathcal{B}(\mathbb{N}, K)}{M}$ is multiplicative.

Definitions and notation: Let $\mathcal{S}=\left(a_{n}\right)_{n \in \mathbb{N}}$ be a coroner sequence. We will denote by $\mathcal{I}(\mathcal{S})$ the ideal of the $f \in A$ such that $f\left(a_{n}\right)=0 \forall n \in \mathbb{N}$. We will denote by $T_{\mathcal{S}}$ the mapping from $A$ into $\mathcal{B}(\mathbb{N}, K)$ which associates to each $f(x)=\sum_{n=0}^{\infty} a_{n} x^{n}$ the sequence $\left(f\left(a_{n}\right)_{n \in \mathbb{N}}\right)$.

Remark: Given a regular maximal ideal $\mathcal{M}=\mathcal{J}(\mathcal{U})$ where $\mathcal{U}$ is thinner than a regular sequence $\mathcal{S}$, then $\mathcal{M}$ contains $\mathcal{I}(\mathcal{S})$.

From [19], (4.6), we have the following theorem: 
Theorem 21: Let $\mathcal{S}$ be a coroner sequence. Then $T_{\mathcal{S}}$ is surjective on $\mathcal{B}(\mathbb{N}, K)$ if and only if the sequence $\mathcal{S}$ is regular.

Theorem 22: Let $\mathcal{S}$ be a regular sequence and let $\mathcal{M}$ be a maximal ideal of $A$. The following two statements are equivalent:

i) $\mathcal{I}(\mathcal{S}) \subset \mathcal{M}$

ii) There exists an ultrafilter $\mathcal{U}$ thinner than $\mathcal{S}$ such that $\mathcal{M}=\mathcal{J}(\mathcal{U})$.

Moreover, the mapping $\Psi$ which associates to each ultrafilter $\mathcal{U}$ thinner than $\mathcal{S}$ the ideal $\mathcal{J}(\mathcal{U})$ is a bijection from $\Sigma(\mathcal{S})$ onto the set of maximal ideals of $A$ containing $\mathcal{I}(\mathcal{S})$.

By Theorems 21 and 22 we have Corollary 22.1

Corollary 22.1: Let $\mathcal{S}$ be a regular sequence. For every maximal ideal $\mathcal{M}$ containing $\mathcal{I}(\mathcal{S})$, the field $\frac{A}{\mathcal{M}}$ is isomorphic to $\frac{\mathcal{B}(\mathbb{N}, K)}{T_{\mathcal{S}}(\mathcal{M})}$.

Theorem 23: Let $\mathcal{S}$ be a regular sequence. For every maximal ideal $\mathcal{M}$ of $A$ containing $\mathcal{I}(\mathcal{S})$, the $K$-Banach algebra quotient norm of $\frac{A}{\mathcal{M}}$ is equivalent to an absolute value extending this of $K$.

Corollary 23.1: For every regular ultrafilter $\mathcal{U}, \mathcal{J}(\mathcal{U})$ is a univalent maximal ideal.

Remark: Consider a maximal ideal $\mathcal{M}$ of $A$ and suppose that there exists $\phi \in \operatorname{Mult}_{m}(A, \|$. $\|)$ which does not lie in the closure of $\operatorname{Mult}_{a}(A, \|$. \|) (which obviously implies that $K$ is not spherically complete). Then $\mathcal{M}$ is not univalent and therefore the $K$-Banach algebra quotient norm of the field $\frac{A}{\mathcal{M}}$ is not equivalent to its norm $\|\cdot\|_{s i}$.

Theorem 24: Let $\mathcal{U}_{1}, \mathcal{U}_{2}$ be two regular ultrafilters. Then $\mathcal{J}\left(\mathcal{U}_{1}\right)=\mathcal{J}\left(\mathcal{U}_{2}\right)$ if and only if $\mathcal{U}_{1}$ and $\mathcal{U}_{2}$ are contiguous.

Corollary 24.1: $\quad$ The relation $\mathcal{R}$ on regular ultrafilters defined as $\mathcal{U}_{1} \mathcal{R} \mathcal{U}_{2}$ if $\mathcal{U}_{1}$ and $\mathcal{U}_{2}$ are contiguous is an equivalence relation whose classes are in bijection with the set of regular maximal ideals of $A$.

Remark: In the general case, Relation $\mathcal{R}$ is not an equivalence relation on a set of filters in $K$. Example: consider a sequence $\left(a_{n}\right)_{n \in \mathbb{N}}$ such that $\left|a_{n}\right|<\left|a_{n+1}\right| \forall n$, put $b_{n}=a_{2 n}$, $c_{n}=a_{2 n+1}$ and consider the filters $\mathcal{F}, \mathcal{G}, \mathcal{H}$ associated to these sequences, respectively. Clearly, both $\mathcal{G}$ and $\mathcal{H}$ are contiguous to $\mathcal{F}$ but $\mathcal{G}$ is not contiguous to $\mathcal{H}$.

\section{Multiplicative norms on $A$}

In order to study more carefully certain multiplicative semi-norms and closed prime ideals, we have to notice a basic theorem: 
Notation: Let $F$ be a field, let $R$ be a commutative $F$-algebra with unity and let $\mathcal{D}$ be a derivation on $R$. Given an ideal $J$ of $R$, we will denote by $\widetilde{J}$ the set of $f \in R$ such that $\mathcal{D}^{(n)}(f) \in J \forall n \in \mathbb{N}$.

Let $\psi \in \operatorname{Mult}(A,\|\|$.$) . We set \operatorname{Subker}(\psi)=\widetilde{\operatorname{Ker}(\psi)}$.

Theorem 25: Let $F$ be a field, let $R$ be a commutative $F$-algebra with unity and let $\mathcal{D}$ be a derivation on $R$. Let $J$ be an ideal of $R$. Then $\widetilde{J}$ is an ideal of $R$ and $(\widetilde{\widetilde{J}})=\widetilde{J}$. Moreover, if $F$ is of characteristic 0 and if $J$ is prime, then so is $\widetilde{J}$.

Since $\left\|f^{\prime}\right\| \leq\|f\| \forall f \in A$, we can derive Corollary 25.1:

Corollary 25.1: $\quad$ Suppose $K$ is of characteristic zero. Let $\mathcal{P}$ be a prime ideal of $A$. Then $\widetilde{\mathcal{P}}$ is a prime ideal of $A$ such that $(\widetilde{\widetilde{\mathcal{P}}})=\widetilde{\mathcal{P}}$. Moreover, if $\mathcal{P}$ is closed, then so is $\widetilde{\mathcal{P}}$.

Corollary 25.2: Suppose $K$ is of characteristic zero. Let $\psi \in \operatorname{Mult}(A, \|$. $\|)$. Then Subker $(\psi)$ is a prime closed ideal.

Theorem 26 shows that $\operatorname{Subker}(\psi)$ is not equal to $\operatorname{Ker}(\psi)$ in the general case. Recall that, given a strictly increasing sequence $\left(r_{n}\right)$ of limit 1 such that $\prod_{n=0}^{\infty}>0$, it is always possible to construct a function $f \in A$ having exactly one zero (of order 1 ) in the circle $C\left(0, r_{n}\right)$ and no other zero. However, due to Lazard's problem, if $K$ is not spherically complete, we can't control the place of these zeroes. With such an element $f$, it is easily proved that $f^{\prime}$ has no zero in the class of $C\left(0, r_{n}\right)$. Theorem 26 is proved by using this property.

Theorem 26: $\quad$ There exist regular maximal ideals $\mathcal{M}$ of $A$ and $f \in \mathcal{M}$, having a sequence of zeroes of order 1 and no other zeroes, such that $f^{\prime} \notin \mathcal{M}$.

In the particular case when $K$ is spherically complete, we can get a more general statement:

Theorem 27: $\quad$ Suppose $K$ is spherically complete and let $\mathcal{M}$ be a regular maximal ideal of $A$. There exists $f \in \mathcal{M}$, having a sequence of zeroes of order 1 and no other zeroes, such that $f^{\prime} \notin \mathcal{M}$.

Remark: When $K$ is not spherically complete, Theorem 26 is less general than Theorem 27 because of Lazard's problem on zeroes of an analytic function [17].

Now we may notice that when the field is of characteristic 2, it is easy to show that for certain maximal ideals $\mathcal{M}$ of $A, \widetilde{\mathcal{M}}$ is not prime. Indeed, by Theorem 26, there exists a coroner maximal ideal $\mathcal{M}$ and $f \in \mathcal{M}$ such that $f^{\prime} \notin \mathcal{M}$. Then $f$ does not belong to $\widetilde{\mathcal{M}}$. Now consider $g=f^{2}$. Then $g^{\prime}=2 f f^{\prime}=0$ hence $g^{(n)} \in \mathcal{M} \forall n \in \mathbb{N}$. If $K$ is of characteristic 3 , we can also construct a similar but less simple counter-example.

Let $\psi \in \operatorname{Mult}(A, \|$. $\|)$ be such that $\psi(P)=\varphi_{\mathcal{F}}(P) \forall P \in K[x]$, with $\mathcal{F}$ a circular filter on $D$ of diameter $r<1$. In [10] it is shown that $\psi$ has a unique continuation to $A$ defined in the same way as $\psi(f)=\varphi_{\mathcal{F}}(f) \forall f \in A$. 
Theorem 28: Let $\psi \in \operatorname{Mult}(A, \|$. $\|)$ satisfy $\psi(P)=\varphi_{\mathcal{F}}(P) \forall P \in K[x]$, with $\mathcal{F}$ a circular filter on $D$ of diameter $r<1$. Then $\psi(f)=\varphi_{\mathcal{F}}(f) \forall f \in A$.

Consequently, all multiplicative norms whose restrictions to polynomials are not the Gauss norm $\|$. I are defined by the circular filters on $D$ of diameter $<1$ and therefore the problem of characterizing continuous multiplicative norms of $A$ only concerns the various continuations of the Gauss norm to $A$.

Theorem 29 shows lets us characterize all continuous multiplicative norms of $A$

Theorem 29: Let $\psi \in \operatorname{Mult}(A,\|\|$.$) be coroner. Then Subker (\psi)$ is not null. Moreover, if $K$ is spherically complete, then, for every $f \in A$ such that $\psi(f)<\|f\|$, there exists $g \in \operatorname{Subker}(\psi)$ admitting no zero which is not a zero of $f$ (the zero of $f$ eventually having a smaller order).

When $K$ is spherically complete, we can adjust the order of zeroes in order that they have slowly increasing orders (in the wide sense), so that the new function we make remains bounded. But any derivation will admit the zeroes of $f$ after certain rank. When the field is not spherically complete, we try to follow a similar way by using Theorem 25.5 [6] in order to obtain a function having some more zeroes than we ask but "the work " of the additional zeroes remains bounded.

Corollary 29.1: Let $\psi \in \operatorname{Mult}(A,\|\|$.$) be coroner. Then \psi$ is not a norm.

Corollary 29.2: Let $\psi \in \operatorname{Mult}(A,\|\|$.$) be a norm. If \psi$ is not $\|$. $\|$, there exists a circular filter $\mathcal{F}$ on $D$, of diameter $r<1$, such that $\psi=\varphi_{\mathcal{F}}$.

Corollary 29.3: Let $\psi \in \operatorname{Mult}(A, \|$. $\|)$ be a norm. If $\psi$ is not $\|$. $\|$, there exists a circular filter $\mathcal{F}$ on $D$, of diameter $r<1$, such that $\psi=\varphi_{\mathcal{F}}$.

On the other hand, each coroner maximal ideal is the kernel of some coroner continuous multiplicative semi-norm of $A$. Consequently:

Corollary 29.4: Let $\mathcal{M}$ be a coroner maximal ideal of $A$. Then $\widetilde{\mathcal{M}}$ is not null.

Concerning the Corona Problem, we may notice this:

Corollary 29.5: $\operatorname{Mult}_{0}(A,\|\|$.$) is included in the closure of \operatorname{Mult}_{a}(A, \|$. $\|)$.

On the other hand, using Theorems 27 and 29, we can prove Theorem 30:

Theorem 30: $\quad$ Let $K$ be spherically complete and $\mathcal{M}$ be a regular coroner maximal ideal. Then $\widetilde{\mathcal{M}}$ is neither null nor equal to $\mathcal{M}$.

Corollary 30.1: $\quad$ Suppose $K$ is of characteristic zero. Then $A$ admits prime closed ideals that are neither null nor maximal ideals. Moreover, if $K$ is spherically complete, then every regular coroner maximal ideal $\mathcal{M}$ of $A$ contains a prime closed ideal $\widetilde{\mathcal{M}}$ that is neither null nor equal to $\mathcal{M}$. 
Remark: The prime closed ideal we construct, which is neither null nor maximal, does not seem to be the kernel of an element of $\operatorname{Mult}(A,\|\|$.$) . Recall that in [2] an example of$ a Banach-K-algebra of analytic elements with no divisors of zero, admitting no continuous multiplicative norm, was constructed.

Now, suppose $K$ is spherically complete. If $\operatorname{Mult}(A,\|\cdot\|)$ only consists of $\operatorname{Mult}_{0}(A, \|$. $\|)$ and $\operatorname{Mult}_{m}(A,\|\cdot\|)$, then $\operatorname{Mult}_{a}(A,\|\|$.$) is dense in \operatorname{Mult}(A,\|\cdot\|)$. Actually, this situation seems much likely.

\section{References}

[1] Berkovich, V. Spectral Theory and Analytic Geometry over Non-archimedean Fields. AMS Surveys and Monographs 33, (1990).

[2] Boussaf, K. and Escassut, A. Absolute values on algebras of analytic elements Annales Mathématiques Blaise Pascal 2, n2, p.15-23 (1995).

[3] Carleson, L. Interpolation by bounded analytic functions and the corona problem Annals of Math. 76, p. 547-559 (1962).

[4] Escassut, A. Spectre maximal d'une algèbre de Krasner, Colloquium Mathematicum, XXXVIII, fasc. 2, p. 339-357, (1978).

[5] Escassut, A. The ultrametric spectral theory, Periodica Mathematica Hungarica, Vol.11, (1), p7-60, (1980).

[6] Escassut, A. Analytic Elements in p-adic Analysis, World Scientific Publishing Inc., Singpore (1995).

[7] Escassut, A. Ultrametric Banach Algebras, World Scientific Publishing Inc., Singapore (2003).

[8] Escassut, A. and Maïnetti, N Spectral semi-norm of a p-adic Banach algebra, Bulletin of the Belgian Mathematical Society, Simon Stevin, vol 8, p.79-61, (1998).

[9] Escassut, A. and Maïnetti, N On Ideals of the Algebra of p-adic Bounded Analytic Functions on a Disk. Bulletin of the Belgian Mathematical Society, Special issue for the Proceedings of the 9-th International Conference on p-adic Functional Analysis.

[10] Escassut, A. and Maïnetti, N About the ultrametric Corona problem Bulletin des Sciences Mathématiques 132, p. 382-394 (2008)

[11] Escassut, A. Ultrametric Corona problem and spherically complete field To appear in Proceedings of the Edinburgh Mathematical Society.

[12] Garandel, G. Les semi-normes multiplicatives sur les algèbres d'éléments analytiques au sens de Krasner, Indag. Math., 37, n4, p.327-341, (1975).

[13] Guennebaud, B. Algèbres localement convexes sur les corps valués, Bull. Sci. Math. 91, p.75-96, (1967).

[14] Guennebaud, B. Sur une notion de spectre pour les algèbres normées ultramétriques, thèse Université de Poitiers, (1973).

[15] Hoffman, K. Banach Spaces of Analytic Functions. Prentice-Hall Inc. (1962). 
(1980).

[16] Krasner, M. Prolongement analytique uniforme et multiforme dans les corps valués complets. Les tendances géométriques en algèbre et théorie des nombres, ClermontFerrand, p.94-141 (1964). Centre National de la Recherche Scientifique (1966), (Colloques internationaux du C.N.R.S. Paris, 143).

[17] Lazard, M. Les zéros des fonctions analytiques sur un corps valué complet, IHES, Publications Mathématiques n14, p.47-75 ( 1962).

[18] Sarmant, M.-C. and Escassut, A. T-suites idempotentes, Bull. Sci. Math. 106, p.289-303, (1982).

[19] Van Der Put, M. The Non-Archimedean Corona Problem Table Ronde Anal. non Archimedienne, Bull. Soc. Math. Mémoire 39-40, p. 287-317 (1974).

Alain Escassut

Laboratoire de Mathématiques UMR 6620

Université Blaise Pascal

(Clermont-Ferrand)

Les Cézeaux

63177 AUBIERE CEDEX

FRANCE

Alain.Escassut@math.univ-bpclermont.fr

Nicolas Maïnetti

LAIC

EA 2146,

IUT, Campus des Cézeaux,

Université d'Auvergne

F-63170 AUBIERE

FRANCE

Nicolas.Mainetti@iut.u-clermont1.fr 